\title{
Un-sounding music; noise is not sound
}

\author{
James Whitehead (JLIAT) \\ SPIEGEL: And what takes the place of philosophy now? \\ HEIDEGGER: Cybernetics. ${ }^{1}$
}

The provisional title for this chapter was "Noise is more than sound," which is unsatisfactory for a number of reasons. Firstly, it can bring to mind the idea of set theory and Venn diagrams with circles labeled "Noise," "Music," and "Sound" in various configurations, allowing various understandings of "noise," "music," or "sound," though always as bounded, finite objects in fixed and finite relationships to one another. Secondly, the framework in which this appears is "Noise in and as Music," which again makes noise into some object, and an object captured "in" music, as if noise is something like a wild animal, a tiger or lion to be caged in a circus. Further, the word "in" is also a culprit, as the big game hunter or specimen collector seeks to put noise into music and noise is effectively tamed or killed in the process. Hence the change in title.

So if noise is not sound, what is it? Two meanings of "sound" are both simultaneously in play here, however now this is not a circle on some diagram, neither is it a beast, nor an object, for I propose that it is not an "it" at all and so even the great metaphysical hunter Heidegger will not find anything, any thing, to cage. (Pun intended.) Noise, noise qua noise, is something like infinity; it is neither fixed nor totalizable. Music's relation to it can be understood as being similar to mathematics and infinity, where infinity is the territory or space that mathematics inhabits. Noise is the territory inhabited

1 Interview given in 1966, quoted in Frank J. Tipler, The Physics of Immortality (London: Macmillan, 1994), 86 . 
not only by music but by all representation, all signification. The inhabitants are finite signifiers, capable of an infinite play of meaning, though they can make no difference to noise's infinite space: subtracting infinity from infinity leaves us with infinity.

\section{Meaning, no Meaning}

We have emerged from a period in the history of theory defined by the linguistic turn, in which definitions play in language games and authorities of meaning, authors, and Gods have been reported dead. Signifiers are arbitrary; grammatical structures collapse and re-form as opposites in texts; musical and artistic structures no longer have meaning but use, a use that has become increasingly entertainment, an entertainment for us. The failure of the philosophy and art of the recent past was the failure to locate any absolute. A continual self-examination, self-doubt, and self-questioning, in which music became a series of experiments, resulted in the abandonment of meaning for the pragmatics of use and a period typified by paranoia of meaninglessness that collapsed into the assured, sensational, empty presence of postmodernity's schizophrenia.

No such disease can be found in science during that period and even through postmodernity, which might account for contemporary philosophy's interest in science as the source of mathematizeable truths. Science did not look inward at its structures but created new ones in its exploration of the great outdoors. ${ }^{2}$ Despite the obvious structures in the great achievement of Western music, these structures were never sufficient, never necessary, and so became a victim of metaphysical critique. ${ }^{3}$ Therefore, it might be argued that,

2 I am referring to relativity theory replacing or adding to Newtonian mechanics, quantum theory, and more recent work on field theory of M-Theory and Strings.

3 For example, "Non-conforming music has no defense against the indifferentism of the mind, that of means without purpose." Theodor Adorno, Philosophy of Modern Music (London: Continuum, 2007), 15. 
from Kant onwards, philosophy led art down some garden path to oblivion. ${ }^{4}$ The problem of meaning in the arts became very personal, to the extent that its present state could be criticized as nothing other than a cult of personality. Being personal for a moment, I did not engage in that. Because of a quite unexpected set of events, I turned my attention to computer science, where definitions are fixed. ${ }^{5}$

\section{“Noise," a Dictionary Definition}

1. a. Sound or a sound that is loud, unpleasant, unexpected, or undesired.

b. Sound or a sound of any kind: The only noise was the wind in the pines.

2. A loud outcry or commotion: the noise of the mob; a lot of noise over the new law.

3. Physics: A disturbance, especially a random and persistent. disturbance, that obscures or reduces the clarity of a signal.

4. Computer science: Irrelevant or meaningless data. ${ }^{6}$

It should be clear that definition 1a allows for an infinite variety of personal expression and opinion, and much has been gained by its exploitation. Postmodern art has been typified by a general destratification, a heterogeneity

4 The path to oblivion or nihilism, the philosophies of Kierkegaard, Nietzsche, Heidegger, Sartre, Camus, et al. It is a nihilism still to be found in contemporary philosophy, for instance in Ray Brassier, Nibil Unbound (Basingstoke: Palgrave Macmillan, 2007). Compare this to science in its predictive achievements: for example, the periodic table as it developed showed clearly where undiscovered elements should fit, and as such their structure and properties could be known before any empirical discovery, one of the high positivist moments in science.

5 No one event in thinking about music and theory made me examine the binary structure of sound files, but a question regarding all pitches combined on an internet board first led me to examine the structure of WAV files in storing sound. A career in computer programming and systems analysis provided the necessary knowledge and access to tools, and then reading the remarkable assertion by Heidegger quoted at the opening of this chapter led me to investigate the world of binary data from a musicological/ philosophical perspective.

6 http://www.thefreedictionary.com/noise, accessed February 20, 2013. 
of "what ever it means to you is what it means." Noise artists are often characterized by or deliberately make themselves predicated on such a definition of noise, and serve to critique and challenge conformity in the arts and society, even to the extent of ironically challenging themselves. Such expressive freedom, for good or bad, rapidly regresses into itself as a kind of noise. Definition 4 is useful as it allows us to absolutize music by showing that a definition of noise is not possible, because no satisfactory containment is possible for a procedure that is necessarily not containable in any finite structure. Such an absolute and deterministic process arises from the nature of noise in computer systems, which are not subject to the inability of fixing a meaning, as occurred in modernity, or to postmodernism's relativism of sensation.

Much of the computer science below is widely known, so I apologize for its pedantic exposition. We do not require a detailed description of the subject except to give us necessary terms for "identity" and "noise" without recourse to esoteric metaphysics or matters of opinion and taste. We need these definitions in order to attempt an objective reexamination of the terms, and from this a radicalizing of the ontology of music.

A simple computer (processor) can only add. It cannot do multiplication, as rather than guess what $7 \times 8$ is, it simply adds up 8 lots of 7 . Furthermore, a simple processor cannot subtract. It achieves subtraction by using complimentary arithmetic, and this process is how a computer system can "identify" or recognize. Complimentary arithmetic only sounds complex. Here is how you compliment a binary number, for example 0100101:

Step 1 . If you see a 0 change it to a 1 and if you see a 1 change it to a 0 . Step 2. Add 1.

That is it. 
So

00100101

Becomes

11011010

Add 1

11011011

Now we can perform subtraction by addition. For example if you want to subtract 37 from 52, depending on how you were taught, you will be carrying and borrowing; you will know how to subtract 7 from 2 and 4 from 5 . You know many rules the computer does not. You can manipulate the digits 0 through 9 . Our processor can only add 0 to 0,1 to 0,0 to 1 , and 1 to 1 . That is all it needs.

Here is the "subtraction" of 37 from 52 using complementary arithmetic:

00110100

$11011011+$

00001111

00001111 is 15 in decimal. If you do not know why or how this worked (and it did; $52-37$ is 15), do not worry; neither does any computer. ${ }^{7}$ When a computer system does something like recognize a face, it (more or less)

7 Why the computer only performs addition and nothing else is part of the "keep it simple stupid" methodology of computing. If you can add, subtract, multiply, and divide simply by addition, then only one circuit for addition is required. Real computer systems may use other additional methods, but they still use Boolean logic that is very simple. Multiple processors and all the other technologies just speed up the process; they do not make it ontologically more sophisticated.

Here is how this "subtraction" is performed: in a 2's Complement number the highest bit is negative.

$\begin{array}{lllllllll}-128 & 64 & 32 & 16 & 8 & 4 & 2 & U & =32+16+4+2=52 \\ 0 & 0 & 1 & 1 & 0 & 1 & 0 & 0 & =-128+64+16+8+2+1=-37 \\ 1 & 1 & 0 & 1 & 1 & 0 & 1 & 1 & =8+4+2+1=15 \\ + & & & & & & & & \end{array}$

In this addition the high bit to the left is "lost." On compact disks and in typical sound files the data is held as signed 16-bit integers. This gives a range of +32767 to -32768 . If a sound file contains a set of numbers all the same, no change in value occurs, which is in effect silence. There are therefore 65,536 possible silences in this format. 
turns the data into binary and subtracts. The logic is simple. If the result of subtracting two numbers is zero, they were the same; any other result tells you they were not the same. This is the cybernetics of identity or recognition!

Often, when you $\log$ in to a computer system, a process needs to check if your user ID is the same as the one stored to allow you access. The system needs to recognize your login identity. Imagine your ID is not something like James123, but "J." The computer subtracts the binary value for "J" from all its stored user IDs until it gets a result of zero. J is stored as 1001010 (ASCII 74), so the subtraction takes place as above. If it subtracts your ID from every ID it has and never gets a zero, it will tell you that you do not exist in its system. For real IDs of more than one character it subtracts each in turn, again "recognizing" your identity when it gets a zero result. Although we seem far from definitions of "noise" and "music," we have now a very simple definition of identity. A thing is identical to itself if its negation added to it leaves nothing. Suddenly Heidegger is back in the room, perhaps.

SPIEGEL: And what takes the place of philosophy now? HEIDEGGER: Cybernetics.

\section{Von Neumann Architecture (or “illegal” operations)}

The Von Neumann Architecture is how modern computers work. One of the features of this architecture is shared memory. The computer's memory (RAM) is used by data, addresses, and instructions. Data is a picture you are drawing, a text file you are editing, a music file, etc. Addresses are pointers the computer uses to find "stuff" in memory and take data there. (Think of an envelope with data inside and an address on the outside.) Instructions tell the computer what to do; they are low-level machine code. For example, "take these bits here, fetch some bits from there." The bits could be data, the here and there places in memory. The point is, rather than have three types of memory — one for data, one for addresses, and one for instructions - they all 
share the same space, for technically good reasons. (Keep it simple, stupid.) Though this simple idea is efficient, problems can occur when data, addresses, and/or instructions get muddled.

A “typical" section of code might look like this:

$\begin{array}{lll}\text { Instruction } & \text { Data } & \text { Address } \\ \text { Do this } & \text { to this } & \text { from here or send it here }\end{array}$

Each would consist of Bytes made of Bits of fixed length (typically 8, 16, or 32).

\section{$0101001010010000 \quad 11100001101010110 \quad 100101010001010$}

However, there is no way of knowing what 11100001101010110 means in isolation. If it is in the first part, it is an instruction; in the middle it is data; and if it is at the end, it is an address. The computer treats whatever arrives in its processing unit as such. If a bit goes missing then

$0101001010010000 \quad 11100001101010110 \quad 100101010001010$

becomes

$$
10100101001000011100001101010110100101010001010 \text { +? }
$$

Now the instruction starts with a 1 and uses the first bit of its data section to complete the instruction. The data now has a missing bit at the beginning and uses the first bit of the address. If the "new" instruction does not exist, 1010010100100001 is not recognized and the computer will not be able to continue. This is an illegal instruction! If the "new" address is not correct, it is an "illegal" address. If the data is altered, then the text file or MP3 or picture 
will have nonsense or rubbish at that place. Here is a simpler example using text.

\section{TAKE THIS HERE}

TAKE $=$ Instruction or operation code

THIS $=$ Data

HERE $=$ An address in memory

We lose the first part of the instruction, so now have:

\section{AKET HISH ERE?}

The $\mathrm{T}$ is lost, but the $\mathrm{T}$ from THIS is used to make up the gap, and so on. AKET is not a task we can perform, HISH is not recognizable data, and ERE? is not a recognizable place. We have nonsense, chaos, noise. This is how errors of "executing data" that are "executing code from a non-executable memory region" can occur.

By way of proof, an actual example. Below, the Microsoft Explorer program crashed trying to write data to a place in memory. This caused an "exception," a problem that the program could not handle, and so the operating systemin this case Windows NT-gave the following message:

Unhandled exception at 0x0a153739 in explorer.exe: 0xC0000005: Access violation writing location 0x090afc40

If we look at the place in explorer.exe where this occurred, we see:

Address OpCode Meaning English

04893739 movd dword ptr [edi+edx],mm2 move some stuff 0489373D psrlq mm2,20h shift some bits left 04893741 movd dword ptr [edi+edx+3],mm2 move some stuff 
The example above shows memory in which an opcode, an instruction, tried to move some data into an area of memory it should not. Either this address in RAM was not there, ${ }^{8}$ or this process (program or application) did not have the rights to perform this move (the location was being used by another process, for instance). Once the context of the bit string is lost, it effectively becomes noise. Such noise causes the program to fail. Coherence, structure, and meaning are lost. ${ }^{9}$

Such a definition of noise from within computer science defines noise as essentially destructive and not as some effect or affect.

\section{The Correlationism of Music}

One of the features of a certain group of contemporary philosopherslabeled variously as Speculative Materialism, Speculative Realism, Object Oriented Philosophy (OOP), or Object Oriented Ontology (OOO)—has been a critique not only of postmodern and continental philosophy but also of Anglo-American analytic philosophies, and the identification of

8 By "not there" I mean that the address generated was larger than the address space of the computer. If you have a street of 100 houses, you cannot send a letter to number 145 .

9 This might clarify some problems with interpretation in the arts. Saussure established that in language signs are arbitrary, and this was further developed by Derrida in Of Grammatology to argue that language worked as a play of differences without any one or set being privileged. There is nothing in the letters "C" "A" "T" that is catty: CAT and DOG could be interchangeable for what they signified. Or in Albanian QEN and MACE: which is CAT and which is DOG? In effect, it has been said we are all speaking "dead languages" (Brassier, Lacan, etc.). There is nothing inside the text to give meaning (and the text here could be an image or a sound structure). Further, it has been argued there is nothing outside of the text that can provide it with a fixed definitive meaning. Famously, "il n'y pas de hors-texte," (Jacques Derrida, of Grammatology (Baltimore, MD: John Hopkins University Press, 1976)), by which there is no arbiter of a fixed and final meaning of a text-neither the author, nor a psychoanalytical or metaphysical theory of interpretation, nor God. "The semantic horizon which habitually governs the notion of communication is exceeded or punctured by the intervention of writing, that is of a dissemination which cannot be reduced to a polysemia. Writing is read, and 'in the last analysis' does not give rise to a hermeneutic deciphering, to the decoding of a meaning or truth" (Jacques Derrida, Limited Inc (Evanston: Northwestern University Press, 1988), 20). 
"correlationism" within all of these philosophies. ${ }^{10}$ Their activity is closely associated with the arts, as was the continental school that they critique. The idea of correlationism effectively critiques all philosophy since Kant, and identifies Kant not as the originator of a Copernican revolution in thought but rather as a reactionary Ptolemaic in his unwillingness and prohibition against thinking "The Real." 11 The justification for this critique is a debate to be had in philosophy and not here. However, I want to employ the same correlationist critique of philosophy to music. Philosophical correlationism can be summarized as the idea that philosophical thought, properly metaphysical thought, never has access to the real, to things in themselves, but it has access to-in fact it is the correlation between-thought and its object. It is only in the correlation that we can ground a philosophical necessity, an absolute and objective knowledge.

Thoughts without content are empty, intuitions without concepts are blind. The understanding can intuit nothing, the senses can think nothing. Only through their unison can knowledge arise. ${ }^{12}$

It is a gross simplification to say we only experience our perception and never what exists outside our perception and therefore cannot know anything of objects but only have knowledge of our experience of them. Key to human experience are Time and Space, but these are, in correlationism, not real

10 This field originated as "Speculative Materialism" from a conference held at Goldsmiths College, University of London, in April 2007. As the numerous titles indicate, the members of that conference (and others) are not so much a "group" or "movement" but instead philosophers who have an interest in a metaphysical realism as critique of the dominant forms of post-Kantian "correlationist" philosophy. For the sake of convenience I use the term "Speculative Materialism" throughout this chapter. The original conference members were Ray Brassier, Iain Hamilton Grant, Graham Harman, and Quentin Meillassoux.

11 '[T] $]$ he central notion of modern philosophy since Kant seems to be that of correlation. By 'correlation' we mean the idea according to which we only ever have access to the correlation between thinking and being, and never to either term considered apart from the other" (Quentin Meillassoux, After Finitude (London: Continuum, 2008), 5).

12 Immanuel Kant, Critique of Pure Reason (London: Penguin, 2007), 86. 
things but merely the necessary constructs for us as humans to experience both the outside world and our inner consciousness.

[T]ime is nothing but the subjective condition under which alone all intuitions can take place within us. ${ }^{13}$

Speculative Materialism wants to reject this correlation in favor of access to the Real, which science seems to have enjoyed, unlike philosophy, since Kant. One of its main motivations is combating the relativism of postmodernity, a relativism similar to the first dictionary definition above of noise as a matter of taste and opinion. ${ }^{14}$ Regardless of philosophical correlationism, it appears so obvious that art exists as a form of correlationism that the idea is often ignored, for correlationism appears to be an a priori necessity that constitutes what art is. One of the consequences of a radical regard to noise and its relation to music is that it exposes and breaks that thought. Alternatively, noise can safely be regarded as one more trope or ingredient for music, perhaps even a dangerous supplement, but one that does not question music's ontology. Crucially, though, if this ontology is questioned then not only is music radicalized but also all the arts. It is from the radicalization of music by noise that a general radicalization of representation might be achieved.

There are numerous theories of art; however, most if not all posit an object and a subject, with the status of the object's art-ness located not in the object but instead in the nature of the relationship between the object and subject. ${ }^{15}$ Music is heard, paintings are seen. Music is played, paintings

13 Kant, Critique of Pure Reason, 69.

14 “[M]athematics' ability to discourse about the great outdoors; to discourse about a past where both humanity and life are absent" (Meillassoux, After finitude, 26).

15 Typically, the subject is human, but this is not necessary. Birds, whales, and other sentient creatures may and probably do engage in music, according to some theories, but the engagement is still essentially the correlation between a subject and an object. Furthermore, musicians have historically found such "musics" of interest, including the more recent genre of music using field recordings. 
are painted. Duchamp's urinal or Cage's silence exist in and because of this correlation. In fact they expose it, rely on it, and work with it. In Duchamp, the context provides the status of art. Cage's 4'33" presents the impossibility of silence as an impossibility for us in our experiential relationship within the performance. Philosophically, the tree falling in the forest may or may not make a sound. No such dilemma exists in 4'33"- - there will always be sound. It is no surprise when Meillassoux posits a time before human thought and a time after. When likewise he proposes existence prior and post Homo sapiens, even the idea of existence and temporality where no cognition exists at all seems acceptable. However, if we move the claim from philosophical ontology to music, we arrive at something quite radical and contrary to the Cageian, correlational ontology of music. Music where no human exists to create the correlation is a very radical idea of music; the idea of non-correlationist music might appear impossible or absurd. We may think of the possibility of noise outside of a human correlation and outside of cognition: it may be debatable but it would not be ontologically impossible, otherwise no debate could take place. Therefore the acceptance of noise as music can either simply ontologize and make noise part of a human correlation or radicalize music itself by destroying its ontology. A choice needs to be made, but before it is, it is crucial to explore just what is at stake.

It would, I think, be difficult to maintain absolutely that sound is only a correlation. To propose that music existed before human thought and will exist after is much more contentious because music is accepted as the correlation in which humans must take a part. ${ }^{16}$ Cage's silence is an impossibility for us, but not an impossibility per se. We have a precise analogy between Kant's

16 Sounds are vibrations and exist in much wider spectra than human hearing. Music may be distinguished only by having air as a medium. Though sounds are found in other media such as water or parts of the electromagnetic spectrum, they are vibrations that are impossible for humans to hear and are independent of any medium. To define sound as only that which humans can hear seems very anthropocentric at minimum and fraught with problems too extensive to explore here. Even if sound is so restricted as to require a medium, "noise" cannot be. Noise occurs in radio transmission and communication and computation. There is cosmic noise that is completely independent of any human correlation. 
phenomena and noumena, the latter existing independently of us yet, for Kant, absolutely removed from us. Cage is a Kantian and the Speculative Materialist would want to challenge this thought. The Speculative Materialist could simply say that it is not at all contradictory to imagine or postulate a time in the far future where no particles vibrated and energy was at its lowest state, so no processing could occur, and that would be a de facto silence. Similar states that are more trivial also exist, for instance at absolute zero. ${ }^{17}$ Therefore, there can be silences, but we can never perceive these because we cannot perceive non-perception. We can think noise outside of perception, outside of a correlation. It exists in any system as an unwanted possibility, which is why noise qua noise is always unwanted even in the arts: it destroys the correlation, or, if used at all, it is used to achieve some destructive act in a very careful way. Noise qua noise is more than a dangerous supplement, for in any correlationist idea of art noise is destructive and fatal unless a limit is applied. The application of a limit renders noise as a token or symbol, for instance of nihilism or anti-art. However noise in-itself is more radical as it effaces the possibility of any symbolism at all (just as the noise in our simple computer system above crashes the system). Noise qua noise is not the elephant in the room but the tiger, and being free in the room and not in some cage (noise as ...), it will devour everybody and everything. What is unwanted in music? What is unwanted is total silence, total chaos, or timelessness (time before and after human life). What is required? Human musicians and audiences, an atmosphere of $78.09 \%$ nitrogen, $20.95 \%$ oxygen, $0.93 \%$ argon, $0.039 \%$ carbon dioxide, and vibrations within this medium in a frequency range of 12 $\mathrm{Hz}$ to $20 \mathrm{kHz}$ and of durations greater than about a second and less than a human lifetime. What this correlation does is make music, as it exists now, an incredibly small fraction of the known universe. There is in principle nothing

17 I can modestly lay claim to 65,536 silences in digital PCM data as stored in computer systems and on compact disks. Any set of data that results in a continuous D.C. offset is, in itself, "silent." See Jliat Still Life \#5: 6 Types Of Silence edition xi, released in 2000. A 10 second compilation of all 65,536 possible silences on audio CD can also be downloaded from http://www.jliat.com/silence/. 
wrong with this, unless any claims are made that this music represents the Real. In the known universe, music as such can only picture the Real as a gross distortion, not withstanding other possible universes and infinities.

Two simple illustrations.

Two American tourists visit Europe and, as they pass from country to country, stay in Holiday Inn hotels where the curtains remain closed and they watch CNN. They return and, though they have visited Europe and can describe it to their fellow Americans, and though we might think they have acted strangely if not stupidly, they have done nothing wrong. They might have noticed the pasta was better in a place called "Italy" - which would be a metaphor for certain desires to use noise in music-but we would not consider that they have experienced or even attempted to experience Europe. A counter argument might be that, as it is impossible to fully experience Europe (or anything), then the action was justified. This would certainly curtail any science, as well as much previous artistic practice, and might be the cost of rejecting or "caging" noise in music. An encounter with Europe would involve an encounter with foreign languages that would be an encounter with noise.

The above is a fiction. A second example is audio CDs. They represent a totalizable set of objects, a set "for humans"-if they were to hear not just that which is familiar- that would consist mostly of unrecognizable noise. ${ }^{18}$

What the loss of the human correlation as music would produce is impossible to define. It is possibly infinite or as large as the known universe and has a temporality of at least trillions of years. How it would be produced

18 "All possible CDs" is a thought experiment. An audio CD stores music by patterns of bits; each audio sample is 16 bits, and each second of sound has 44,100 samples, so 16 x 44,100 gives us a second of sound. Multiply by two for stereo, and then by 60 for a minute, then by 74 for the old specification of the maximum duration in minutes of an audio $\mathrm{CD}$. (The fact you can get longer and different formats is for my purpose irrelevant here.) Multiply 16 × 44,100 x 2 × 60 × 74 and we get 6,265,728,000 bits. What follows is that, in this $\mathrm{CD}$ format, there are $2^{6265728000}$ possible CDs, and no more. Much of this universe would appear for us as noise, yet "noise" would be more representative of its reality. 
and perceived or known is likewise not limited. The alternative I can predict with certainty: if noise is made into music it will become sound, vibrations in air of given frequencies and given length made for humans and in the main by humans. ${ }^{19}$

From the brief description of computation we can use a definition to identify music trivially_- A thing is identical to itself if its negation added to it leaves nothing." However, also from computer science, the definition of noise precludes its definition, as "noise causes the program to fail." Computer science defines noise as essentially destructive: coherence, structure and meaning are lost, and with noise so too is the possibility of identification, limit, boundaries, and rules. This may not be wished for, wanted or liked, but it represents the Real in non-correlationist terms. Noise is the Real.

\section{Size Matters}

One of the impetuses behind the current philosophical thinking, which is associated with the arts in the form of Speculative Realism and Object Oriented Ontology, is the critique in the sciences of an egocentric universe (one more step from a logocentric, and phallo-logocentric universe of the previous philosophemes). Meillassoux and Brassier note that science has revealed a universe far larger than humanity, one 300 billion years old with

19 This includes not only music with animals as sources—-for instance, whale song mediation discs—but also objects such as computer generated jazz (e.g., http://www.youtube.com/watch?v=P-Sjgn78rgw) or generative music in general. For example, "Tiklbox composes an endless stream of high-quality beautiful ambient music ideal for relaxation, reflection, meditation \& quiet contemplation" (Intermorphic: play with ideas, accessed February 28, 2013, http://www.intermorphic.com/). Or, similarly, "Scape makes music that thinks for itself. From Brian Eno and Peter Chilvers, creators of Bloom, Scape is a new form of album which offers users deep access to its musical elements. These can be endlessly recombined to behave intelligently: reacting to each other, changing mood together, making new sonic spaces. Can machines create original music? Scape is our answer to that question: it employs some of the sounds, processes and compositional rules that we have been using for many years and applies them in fresh combinations, to create new music. Scape makes music that thinks for itself" (Brian Eno and Peter Chilvers, Generative Music, accessed March 1, 2013, http://www.generativemusic.com/). 
a future of trillions of years. ${ }^{20}$ The contemporary age has, in many spheres, expanded beyond human perception if not comprehension. Trivially, in music technology the 45-rpm record dictated the length of popular songs, the LP the "concept" album. Such domestic realities also played a part in structuring classical music through the length of operas or concertos, similar to the reasons Dutch paintings of the 17 th and 18th centuries were made small enough to fit into the houses of the Dutch bourgeoisie, unlike the majestic canvasses of the Louis XIV epoch in France or the massive abstract expressionist paintings of the large lobbies of Manhattan office buildings in the last century. ${ }^{21}$ However, just as the trillion escapes us, so does the presentday storage capacity of the media around us. It is not unusual to have in a home terabytes of storage, or on these devices music, which, if it has not already, will soon assume a temporality greater than that of any potential listener's life expectancy. The same could be said of other media, text, or movies, but it is the ubiquity of the mobile phone and musical playlists that offers the challenge: no one can listen to it all. And if it escapes our listening, it breaks the correlationist grip in a practical demonstration of a real that is bigger than ourselves, bigger than human perception. We already know that sound shares characteristics with a larger electromagnetic spectrum, and over 100 years ago synesthesia was an important influence in the development of a modern art that envisaged a unification of meaning across forms, but

20 Trillion is the new million! The recent economic crisis has exposed this inhuman number. It is possible to live long enough to count to a million, even a billion, but not a trillion.

21 "Sony had initially preferred a smaller [audio CD standard] diameter, but soon after the beginning of the collaboration started to argue vehemently for a diameter of $120 \mathrm{~mm}$. Sony's argument was simple and compelling: to maximize the consumer appeal of a switch to the new technology, any major piece of music needed to fit on a single CD ... Beethoven's Ninth Symphony was quickly identified as the point of reference-according to some accounts, it was the favorite piece of Sony vice-president Norio Ohga's wife. And thorough research identified the 1951 recording by the orchestra of the Bayreuther Festspiele under Wilhelm Furtwängler, at seventy-four minutes, as the slowest performance of the Ninth Symphony on record. And so, according to the official history, Sony and Philips top executives agreed in their May 1980 meeting that 'a diameter of 12 centimeters was required for this playing time." Tim Büthe and Walter Mattli, The New Global Rulers: The Privatization of Regulation in the World Economy (Princeton: Princeton University Press, 2011), 46. 
this was always "for us," it never escaped us. And when science did escape our common sense and human world, it left a kind of alienation in being as expressed in the arts, ${ }^{22}$ or worse a return to a fundamentalism. So a symphony orchestra appears to operate by some laws that are more sharia than scientific.

There are works that challenge human temporality in listening. Amongst others the artist Mattin's recent work ${ }^{23}$ comprises more than 40 hours of MP3 recordings, and there are and have been numerous practices that challenge accepted conventions of musical forms and durations. ${ }^{24}$ However, these works in the main remain correlational in an explicit or implicit "human" correlation, by which they work as art. Temporality normally corrals music into what is "listenable" and regards anything outside this as outside of a possible listening experience, as data that can provide us with no meaning, as noise. Working with temporality in music as a "real" timescale, not merely human timescales, would require a radicalization of musical form even if it were still limited to sound. We might have to abandon the correlationism of listening, playing, and making for other methods. These are now available. ${ }^{25}$

22 See the influential book The Two Cultures by C.P. Snow (London: Cambridge University Press, 2001).

23 IMPROKUP! Improvisation as squatting and living together (DVD and booklet, w.m.o/r 38, Stockholm, 2012)

24 For example, ASLSP (As SLow aS Possible) by John Cage, a performance of which at St. Burchardi church in Halberstadt, Germany, is projected to last 639 years; Longplayer by Jem Finer, which is designed to last for one thousand years; or the prolific output of noise artists such as Merzbow. There are also examples of "non-sonic music" in the work of Yoko Ono, Karlheinz Stockhausen, and recently Seth KimCohen and numerous others. However, the idea of sound, human temporality, and music in some form of correlation still predominates and limits the art form.

25 MP3 is a method of compressing sound files. It is "lossy" in that parts of the original sound are lost during compression. What makes working with the MP3 format special is not this feature, but instead that, unlike other formats (WAV, MP4, etc.), MP3 files can be spliced together as larger entities. The ability to combine these in a quick and simple way makes the creation of large structures very simple, almost like the process of DNA synthesis. At its most simple, if you have a folder containing some MP3 files, in Microsoft Windows using the DOS Copy command it is possible to concatenate these into one large and playable file. To do this, open the MS-DOS shell, and in the MP3 folder type "Copy /b *.MP3 big.MP3" (without quotes). Big.MP3 will now contain all the other MP3s in one playable file. It is then simple to create tools with which it is possible to generate and manipulate sound files of lengths impracticable to work with in real time (real time here being real time for humans); see, for example, http://www.jliat.com/1tb/. 
It might be that such realities can be experienced only conceptually and imaginatively, only in the imagination that such territories can be traversed. Today I can hold in the palm of my hand sufficient storage capacity for more than seven years of music. ${ }^{26}$ Ten of these devices would store an un-listenable quantity — yet one that is by all means an objective reality—and the data that exists in data farms far exceeds any single human experience. ${ }^{27}$

Do we regard this externality as unreal for us, as not sensible, and in its incomprehensibility as noise, or can we find other methodologies for the appreciation of spaces and sizes that are greater than the human? Science has already done this, and the philosophies above attempt the same. The philosopher Laruelle also has a "Real" that is seemingly limited to the human, yet employs a generic matrix that is infinite and incomprehensible. ${ }^{28}$ Meillassoux's contingent hyper-chaos, which offers a potential exo-human infinity in his subsequent work, reverts this back to the human scale of possibilities as the source of some future grieving deity; ${ }^{29}$ however, the hyper-

26 See, e.g., http://www.seagate.com/gb/en/internal-hard-drives/desktop-hard-drives/desktop-hdd/, accessed February 23, 2013.

27 The world's total storage capacity is obviously large and expanding, and difficult to estimate, but as of 2011 one estimate gives 295 exabytes. (1,000,000 terabytes $=1$ exabyte. $)$ Therefore, that is, in 2011, 295 million years of potential sound in MP3 format. If this all seems a little unrealistic or crazy, it could be because it is, or it could be that we somehow cannot or do not want to comprehend the Real, or what Meillassoux calls The Great Outdoors. If art is to get real, it has to work with these scales, because they are there (Lucas Mearian, "Scientists calculate total data stored to date: 295+ exabytes," Computer World, February 14, 2001, accessed February 23, 2013, http://www.computerworld.com/s/article/9209158/ Scientists_calculate_total_data_stored_to_date_295_exabytes).

28 Laruelle's work with Non-Philosophy offers an alternative approach to radicalizing music, for instance in the idea of "small thoughts, everywhere and with every individual" (John Mullarkey and Paul Smith, Laruelle and Non-Philosophy (Edinburgh: Edinburgh University Press, 2012), 1). Briefly, the "Non" here is not a negation but similar to the Non in Non-Euclidian geometry, which "opened up" mathematics. Jarrod Fowler has developed from this his concept of Non-Musicology (see http://www.nonmusicology.com/). "Non-Euclidean' became a by word for non-absolute knowledge ... Even the concept of truth was not absolute ... Mathematics was open-ended, uncompletable, infinite ..." (John Barrow, The Book of Nothing (London: Jonathan Cape, 2000), 157). Music once had a mathematical (Pythagorean) ontology; if this were regained it could likewise become an infinite object.

29 The necessity of contingency (Meillassoux's proposal in After Finitude) is employed as an ethical resource proposing a future deity who can effectively (bring justice) mourn victims of cruelty who have died and not been mourned. Quentin Meillassoux, "Spectral dilemma," Collapse vol. IV: Concept Horror, Robin Mackay, ed., December 2012. 
chaos generated by the necessity of contingency is not limited to or for the human. ${ }^{30}$ Speculative Materialist philosophies might, in the allure of nontotalizable objects, appear as philosophemes of the Romantic poets, as if the incomprehensibility of these objects were not available to the philosophic mind still bound by a correlationism, or nihilism of life as an existential being. The problematic for philosophers and artists, including musicians, seems to lie in their gaze, ${ }^{31}$ a problematic of Petrarch's mountain ${ }^{32}$ in looking in the wrong direction into the nature of the subject/object relationship and not into the reality of the object. However, it is now possible to make works of sound that might be considered music even if they are un-listenable not only in practice but also in principle. A simple reaction is to reject this and go back to the cloisters of a medieval universe of music where man is, and always is, at its center. But this is now only an illusion. If noise qua noise does come into music as anything more than another trope, it exposes the Real, expanding music beyond the human; how we do this, understand it, appreciate it, or disseminate it is already given by technology.

\section{For Want of a Conclusion}

Through the advent of a cybernetics of sound-which generalizes, democratizes, and personalizes music in MP3 playlists on mobile phones, hard drives, and the internet-music, more than any other medium, has direct access to hyper-objects, hyper-chaos, territories finitely and possibly infinitely bigger than the human, which is noise as un-sounding music.

30 "Our absolute, in effect, is nothing other than an extreme form of chaos, a hyper-Chaos, for which nothing is or would seem to be impossible, not even the unthinkable.... [F]ar from guaranteeing order, it guarantees only the possible destruction of every order," Meillassoux, After Finitude , 64.

31 In this case non-Lacanian.

32 On April 26, 1336, Petrarch climbed to the top of Mont Ventoux from where he could see the Alps. The act is often used as a metaphor for the turning point in which medieval thought is re-directed outward to the real world of nature as opposed to the cloistered inner world of the "Dark Ages," a term of which Petrarch is credited with first use. 
However, strong forces counter the ideas expressed above of removing the perceptual core of music's ontology. These ideas can be dismissed as fringe phenomena, as peculiar and invalid ideas because they fail to account for the general consensus that music is predicated on sounds that are heard, that the "real" world of music is listening, that the idea of a non-correlationist Real is contradicted by the reality of listening in the "real" world of music. However, this is not the case. The quantities of data in volumes located on YouTube, Bandcamp, SoundCloud, and elsewhere, though created almost certainly with the intention to be heard, cannot in fact be heard. Human perceptual systems can no longer cope with the scale of these quantities. Any attempt at perception will be fractional, distorted, and always incomplete. Overwhelming the perceptual system effaces communication. This has already occurred. And once quantity overwhelms the system, qualitative judgments are no longer possible. ${ }^{33}$ And this has also occurred. Within the world of music, mediators of value and taste have changed from the few to the many, and quantity effaces any qualitative ability. In general, everything published on the web is "Awesome!" Obviously this is not immediately good news for anyone who thinks music has a value, or wants to produce value from it. It is a problem for manufactures and retailers of music as product, for reviewers of music and arbiters of taste, and perhaps even for musicological study and evaluation in academic institutions. ${ }^{34}$ What has happened to music? Is it now beyond good and evil?

And do you know what "the world" is to me? Shall I show it to you in my mirror? This world: a monster of energy, without beginning, without end... ${ }^{35}$

33 This use of noise to overwhelm a system is found in nature and in the military. Herding animals confuses the predator by overwhelming its perceptual ability to make a judgment.

34 This might also represent a problem for the "artist" as some lone Nietzschean Übermensch or Romantic genius, a problem for the artist as anything other than yet another of the numberless pop wannabes?

35 Frederick Nietzsche, Will to Power (New York: Vintage Books, 1967), 549. 


\section{Alice Kemp (Germseed)}

\section{What is noise (music) to you?}

I'll keep this personal and not wander into histories or hair-splitting genre definitions. Noise is a living entity, pure expression, force, simple and complex. It is a system of diametrical differences-it can be a cycle of giving and receiving, an exchange of power. It is chaos racket or absolute laser precision. It is extended drone and fragmentary bursts. It can deal in specifics or unknowns. It can be both irritant and balm, purgative, cleansing, medicinal. A source of both exhilaration and panic, fight and/or flight, control and release. It can be what you need to escape from, whilst being just exactly what you need. It can act as social cohesion, compelling, repellent, tribal, or hermitic. It can be deftly brutal or subtlety incarnate. Absurd, perfect slapstick, funny haha, deadpan serious. It can contain portals that allow access to altered states. Crucially, silence can be noise (and again, vice versa).

\section{Why do you make it?}

Seduced by soundworlds since my early years, I work with these ideas because of their very existence. 